\title{
INEQUALITIES IN DIMENSION THEORY FOR POSETS
}

\author{
WLLIAM T. TROTTER, JR.
}

ABSTRACT. The dimension of a poset $(X, P)$, denoted $\operatorname{dim}(X, P)$, is the minimum number of linear extensions of $P$ whose intersection is $P$. It follows from Dilworth's decomposition theorem that $\operatorname{dim}(X, P) \leq$ width $(X, P)$. Hiraguchi showed that $\operatorname{dim}(X, P) \leq|X| / 2$. In this paper, $A$ denotes an antichain of $(X, P)$ and $E$ the set of maximal elements. We then prove that $\operatorname{dim}(X, P) \leq|X-A| ; \operatorname{dim}(X, P) \leq 1+$ width $(X-E)$; and $\operatorname{dim}(X, P) \leq 1+2$ width $(X-A)$. We also construct examples to show that these inequalities are sharp.

1. Introduction. Dushnik and Miller [4] defined the dimension of a poset, denoted $\operatorname{dim}(X, P)$ or $\operatorname{dim} X$, to be the minimum number of linear extensions of $P$ whose intersection is $P$. Equivalently, Ore [7] defined $\operatorname{dim}(X)$ to be the smallest integer $k$ such that $(X, P)$ is isomorphic to a subposet of $R^{k}$. We refer the reader to [1], [2], and [8] for other definitions and preliminaries. In this paper we establish inequalities involving dimension, width, height, and cardinality. A number of such inequalities are known and we begin by stating a sampling of them.

Theorem. For any posets $X, Y$, any chain $C \subseteq X$, and any point $x \in X$, the following inequalities bold.

(1) $\operatorname{dim}(X-x) \leq \operatorname{dim} X \leq 1+\operatorname{dim}(X-x)$ [5], [1],

(2) $\operatorname{dim} X \leq 2+\operatorname{dim}(X-C)[5]$,

(3) $\operatorname{dim} X \leq$ width $X[5]$,

(4) $\operatorname{dim} X \leq|X| / 2$ (Hiraguchi's theorem [5], [1]),

(5) $\operatorname{dim}(X \times Y) \leq \operatorname{dim} X+\operatorname{dim} Y$.

A poset has dimension one iff it is a chain. If a poset consists of an antichain of at least two points, then its dimension is two. Throughout the remainder of this paper we will assume that $X$ is a poset which is neither a chain nor an antichain. We will use the symbols $A$ and $E$ to denote an arbitrary antichain in $X$ and the set of maximal elements respectively. If

Received by the editors July 6, 1973.

AMS (MOS) subject classifications (1970). Primary 06A10, 05A20.

Key words and phrases. Poset, dimension, irreducible. 
$|X-A|=1$, but $X$ is not a chain, then it is trivial to show that $\operatorname{dim} X=2$. Therefore we will assume that for any antichain $A \subseteq X,|X-A| \geq 2$. Furthermore we do not distinguish between a poset and'its dual.

2. Some new inequalities. In this section we establish some new inequalities for the dimension of a poset.

Lemma 1. Suppose $x$ and $y$ are incomparable points in a poset $X$, but for every $z \in X-\{x, y\}, z>x$ iff $z>y$ and $z<x$ iff $z<y$. Then $\operatorname{dim}(X-x)$ $=\operatorname{dim} X$ unless $X-x$ is a chain.

Proof. If $X-x$ is not a chain then $\operatorname{dim} X-x \geq 2$; let $L_{1}, L_{2}, \cdots, L_{t}$ be linear extensions of $P \mid X-x=P^{\prime}$ whose intersection is $P^{\prime}$. In $L_{1}, L_{2}, \cdots, L_{t-1}$ insert $y$ immediately over $x$, and in $L_{t}$ insert $y$ immediately under $x$. The resulting linear extensions of $P$ intersect to give $P$, and thus $\operatorname{dim} X \leq \operatorname{dim} X-x$. We note that if $X-x$ is a chain, then $\operatorname{dim} X-x=\operatorname{dim} X-y=1$, but $\operatorname{dim} X=2$.

A trivial modification of this argument also proves the following statement.

Lemma 2. Suppose $x>y$ in $P$ but for every $z \in X-\{x, y\}, z>x$ iff $z>y$ and $z<x$ iff $z<y$. Then $\operatorname{dim} X=\operatorname{dim} X-x=\operatorname{dim} X-y$.

Lemma 3. If $|X-A|=2$, then $\operatorname{dim} X=2$.

Proof. We may assume without loss of generality that $X$ cannot be reduced by either of the preceeding lemmas to a poset with the same dimension as $X$ by having fewer number of points. Then it is easy to see that $X$ is isomorphic to a subposet of one of the following posets.
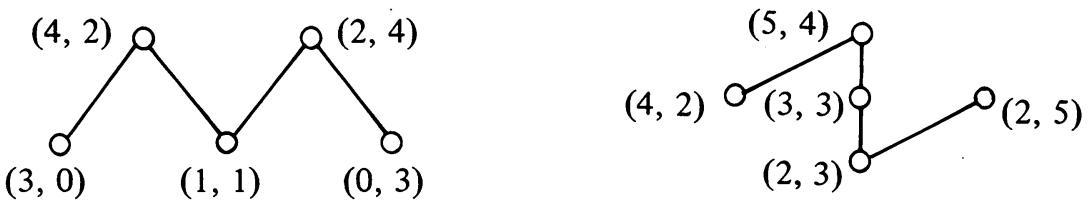

Figure 1

But the coordinatizations given in Figure 1 show that each of these has dimension 2 . 
Since the removal of a point cannot decrease the dimension more than one, we have proved the following result.

Theorem 2. If $|X-A| \geq 2$, then $\cdot \operatorname{dim} X \leq|X-A|$.

Combining this result with the easily obtained bound $\operatorname{dim} X \leq$ width $(X)$, we have established Hiraguchi's theorem ${ }^{1}$ that $\operatorname{dim} X \leq|X| / 2$ when $|X| \geq 4$.

We also note that the standard examples of maximal dimensional posets, denoted $S_{n}^{0}[2],[8]$, show that the bounds $\operatorname{dim} X \leq$ width $(X), \operatorname{dim} X \leq|X-A|$ and $\operatorname{dim} X \leq|X| / 2$ are best possible.

Theorem 3. $\operatorname{dim} X \leq$ width $(X-E)+1$.

Proof. Let $t=$ width $(X-E)$; then by Dilworth's theorem [3], there is a partition $X-E=C_{1} \cup C_{2} \cup \cdots \cup C_{t}$, where each $C_{i}$ is a chain. For each $i$, let $L_{i}$ be a linear extension of $P$ which is a lower extension [1] with respect to $C_{i}$. Form a linear extension $L_{t+1}$ of $X$ by placing all maximal elements on top of some linear extension $M$ of $X-E$ and then ordering the maximal elements in $L_{t+1}$ in the reverse order imposed on them by $L_{t}$. It is easy to see that $L_{1} \cap L_{2} \cap \cdots \cap L_{t+1}=P$, and the proof of our theorem is complete.

For $w=1$ and $w=2$, the following examples show that the bound is best possible.
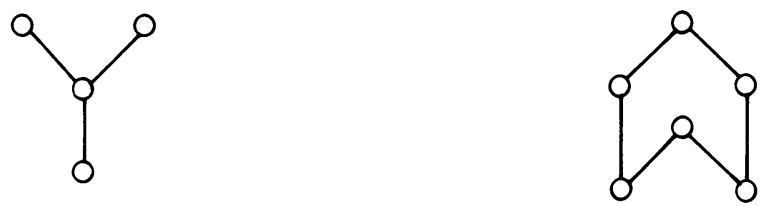

Figure 2

For $n \geq 3$, we construct a poset $Y_{n}$ as follows. $Y_{n}$ has $3 n+2$ points $\left\{a_{1}, a_{2}, \cdots, a_{n}, a_{n+1}\right\} \cup\left\{y_{1}, y_{2}, \cdots, y_{n}\right\} \cup\left\{x_{1}, x_{2}, \cdots, x_{n}\right\} \cup\{p\}$. The points $\left\{a_{i} \mid i \leq n\right\},\left\{y_{i} \mid i \leq n\right\}$ form a copy of $S_{n}^{0}$. Each $y_{i}$ covers $x_{i}$; $p$ covers $a_{1}, a_{2}, \cdots, a_{n}$ but $p I a_{n+1}$; and $a_{n+1}$ covers all $x$ 's. We illustrate this construction with the Hasse diagram for $Y_{3}$.

${ }^{1} \mathrm{~K}$. P. Bogart first suggested that an elementary proof of Hiraguchi's theorem might be produced by considering the complement of the largest antichain. R. Kimble has independently discovered this result; his proof will appear in his thesis [6]. 


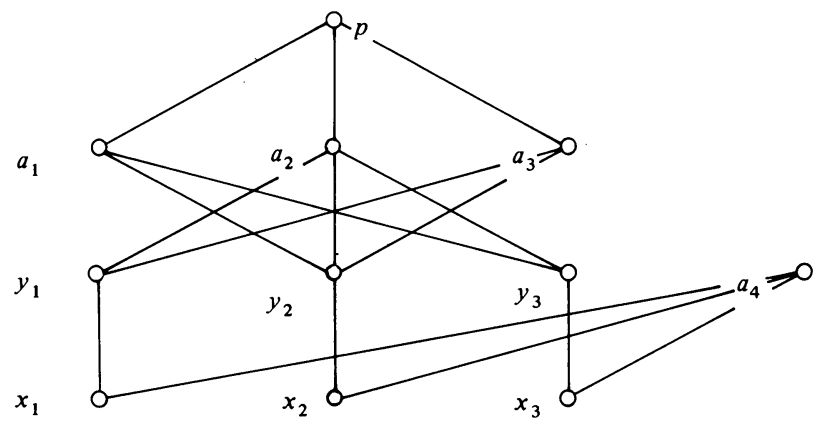

Figure 3

It is clear that if $E=\left\{p, a_{n+1}\right\}$, then $w\left(Y_{n}-E\right)=n$. We now show that $\operatorname{dim} Y_{n}=n+1$.

Suppose $\operatorname{dim} Y_{n} \leq n$; let $L_{1}, L_{2}, \cdots, L_{n}$ be linear extensions of $Y_{n}$ whose intersection is the partial ordering on $Y_{n}$. We may assume that the $L$ 's have been numbered so that $x_{i}$ is over $a_{i}$ in $L_{i}$. Now $a_{n+1}$ is over all $x$ 's; since $y_{i} I a_{n+1}$ but $y_{i}<a_{j}$ for all $j \neq i, j \leq n, y_{i}$ is under $a_{n+1}$ in all lists except possibly $L_{i}$. Hence we must have $y_{i}$ over $a_{n+1}$ in $L_{i}$. Since $p>y_{i}$ for all $i$, this implies $p$ is over $a_{n+1}$ in every $L_{i}$. The contradiction shows that $\operatorname{dim} Y_{n}=n+1$.

We note that it is straightforward to show that each $Y_{n}$ is irreducible; i.e., the removal of any point from $Y_{n}$ lowers the dimension to $n$. We refer the reader to [9] for details.

Theorem 4. $\operatorname{dim} X \leq 2$ width $(X-A)+1$.

Proof. Suppose $t=$ width $(X-A)$ and let $X-A=C_{1} \cup C_{2} \cup \cdots \cup C_{t}$ be a decomposition into chains. For each $i$, let $L_{2 i-1}$ and $L_{2 i}$ be upper and lower extensions, respectively, of $C_{i}$. Then let $M$ be an ordering of $A$ which is the reverse of ordering imposed on $A$ by $L_{2 t}$; then let $L_{2 t+1}$ be any linear extension of $P$ whose restriction to $A$ is $M$. Clearly $L_{1} \cap L_{2} \cap \cdots \cap L_{2 t+1}=P$ and the proof of our theorem is complete.

To show that the inequality of Theorem 4 is best possible, we construct for each $n \geq 1, b \geq 1$ a poset $X(n, b)$ as follows. $X(n, b)$ contains a maximal antichain $A$, and $X(n, b)-A=X_{U} \cup X_{L}$ is the natural decomposition into upper and lower halves. $X_{U}$ and $X_{L}$ each consist of $n$ incomparable chains with each chain containing $b$ points. Every point in $X_{U}$ is greater 
than every point in $X_{L}$. For each ordered pair $(S, T)$ where $S$ is an order ideal of $\hat{X}_{U}$ and $T$ is an order ideal of $X_{L}$, there is a point in $A$ which is less than all points in $S$ and greater than all points in $T$. We illustrate this definition with the Hasse diagrams for $X(1,2)$ and $X(2,1)$.
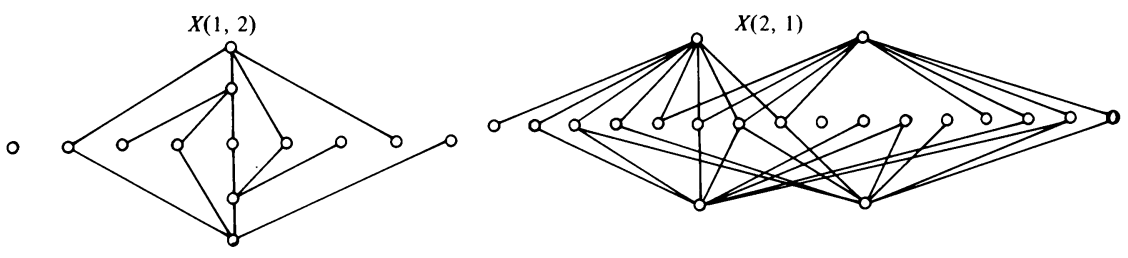

Figure 4

We note that the width of $X(n, b)-A$ is $n$. However, it can be shown [10] that for sufficiently large $b, \operatorname{dim} X(n, b)=2 n+1$.

3. Some open problems. Al though we have outlined in this paper an elementary proof of Hiraguchi's theorem: $\operatorname{dim} X \leq|X / 2|$, it is not known whether or not every poset contains a pair of points whose removal lowers the dimension at most 1 .

A second problem involves cartesian products. Although $\operatorname{dim}(X \times Y)$ $\leq \operatorname{dim} X+\operatorname{dim} Y$, it is easy to construct posets $X, Y$ for which $\operatorname{dim}(X \times Y)$ $<\operatorname{dim} X+\operatorname{dim} Y$. (In fact $\operatorname{dim}\left(S_{n}^{0} \times S_{n}^{0}\right) \leq 2 n-2$.) The question involves the accuracy of the lower bound $\operatorname{dim}(X \times Y) \geq \max \{\operatorname{dim} X, \operatorname{dim} Y\}$.

\section{REFERENCES}

1. K. Bogart, Maximal dimensional partially ordered sets. I, Di screte Math. 5 (1973), 21-32.

2. K. Bogart and W. Trotter, Maximal dimensional partially ordered sets. II, Discrete Math. 5 (1973), 33-44.

3. R. P. Dilworth, A decomposition theorem for partially ordered sets, Ann. of Math. (2) 51 (1950), 161-166. MR 11, 309.

4. B. Dushnik and E. Miller, Partially ordered sets, Amer. J. Math. 63 (1941) 600-610. MR 3, 73.

5. T. Hiraguchi, On the dimension of orders, Sci. Rep. Kanazawa Univ. 4 (1955), 1-20. MR 17, 10.45; 19, 1431.

6. R. Kimble, Ph.D. Thesis, M.I.T., Cambridge, Mass.

7. O. Ore, Theory of graphs, Amer. Math., Soc. Colloq. Publ., vol. 38, Amer. Math. Soc., Providence, R.I., 1962. MR 27 \#740.

8. W. T. Trotter, Dimension of the crown $S_{n}^{k}$, Discrete Math. 8 (1974), 85-103. 
9. W. T. Trotter, Some families of irreducible partially ordered sets, U.S.C. Math. Tech. Rep. 06A10-2, 1974.

10. - Irreducible posets with large height exist, J. Combinatorial Theory Ser. A 17 (1974).

DEPARTMENT OF MATHEMATICS, UNIVERSITY OF SOUTH CAROLINA, COLUMBIA, SOUTH CAROLINA 29208 